\title{
A Retinotopic Attentional Trace after Saccadic Eye Movements: Evidence from Event-related Potentials
}

\author{
Durk Talsma $^{1,2 *}$, Brian J. White ${ }^{3 *}$, Sebastiaan Mathôt ${ }^{2,4,5}$, \\ Douglas P. Munoz ${ }^{3}$, and Jan Theeuwes ${ }^{2}$
}

\begin{abstract}
Saccadic eye movements are a major source of disruption to visual stability, yet we experience little of this disruption. We can keep track of the same object across multiple saccades. It is generally assumed that visual stability is due to the process of remapping, in which retinotopically organized maps are updated to compensate for the retinal shifts caused by eye movements. Recent behavioral and ERP evidence suggests that visual attention is also remapped, but that it may still leave a residual retinotopic trace immediately after a saccade. The current study was designed to further examine electrophysiological evidence for such a retinotopic trace by recording ERPs elicited by stimuli that were presented immediately after a saccade $(80 \mathrm{msec}$
\end{abstract}

SOA). Participants were required to maintain attention at a specific location (and to memorize this location) while making a saccadic eye movement. Immediately after the saccade, a visual stimulus was briefly presented at either the attended location (the same spatiotopic location), a location that matched the attended location retinotopically (the same retinotopic location), or one of two control locations. ERP data revealed an enhanced P1 amplitude for the stimulus presented at the retinotopically matched location, but a significant attenuation for probes presented at the original attended location. These results are consistent with the hypothesis that visuospatial attention lingers in retinotopic coordinates immediately following gaze shifts.

\section{INTRODUCTION}

As first proposed in a classic essay by Walls (1962), eye movements evolved as a means of gaze stabilization. When we shake our head in disagreement, our eyes move so that we are able to maintain fixation. When we look out of the window of a moving train, our eyes automatically track the passing landscape. These types of gaze-stabilizing reflexes cancel out over $90 \%$ of the retinal image motion that would otherwise occur (Ferman, Collewijn, Jansen, \& Van Den Berg, 1987; Slavenski, Hansen, Steinman, \& Winterson, 1979), thus shielding us from a loss of visual acuity and sensitivity (Murphy, 1978; Westheimer \& McKee, 1975). Paradoxically, however, eye movements are also the source of the most violent disruptions of visual stability. Yet, as we scan our visual surroundings, we perceive neither a disruption nor a mismatch between what we see before and after such a saccade. In fact, we are only dimly aware that we make saccades at all, even when executing visually guided movements (Cameron, Enns, Franks, \& Chua, 2009).

The question of how we are able to maintain visual stability in the face of continuous eye movements and corresponding retinal changes has intrigued philosophers

\footnotetext{
${ }^{1}$ Ghent University, Belgium, ${ }^{2}$ Vrije Universiteit, Amsterdam, The Netherlands, ${ }^{3}$ Queens University, Kingston, Canada, ${ }^{4}$ Aix-Marseille Université, ${ }^{5}$ Le Centre National de la Recherche Scientifique, France *These authors contributed equally to this study.
}

for centuries (Grüsser, 1986; see also Melcher, 2011, for a recent review), but striking advances have been made in recent years. Many researchers believe that there is a "predictive remapping" mechanism that actively compensates for the retinal displacements caused by eye movements. In a landmark paper, Duhamel, Colby, and Goldberg (1992) used remapping to refer to the striking changes in the receptive field properties of neurons in the monkey parietal cortex (see also Wurtz, 2008; Sommer \& Wurtz, 2006; Nakamura \& Colby, 2002). They found that many neurons become responsive to the part of the visual field that will be brought into their receptive field by an impending saccade. In a sense, these neurons simulate the retinal changes that a saccade will bring about just before the eyes actually move. Duhamel and colleagues (1992) speculated that such predictive (i.e., presaccadic) remapping could underlie visual stability across eye movements. This hypothesis has inspired many researchers to use ERP (Parks \& Corballis, 2008, 2010) and behavioral (Hunt \& Cavanagh, 2011; Rolfs, Jonikaitis, Deubel, \& Cavanagh, 2011; Mathôt \& Theeuwes, 2010a; Melcher, 2007) measures to investigate predictive remapping indirectly. Although translating neurophysiological results to predictions about behavior is not straightforward, several behavioral studies (Harrison, Mattingley, \& Remington, 2012; Rolfs et al., 2011; Mathôt $\&$ Theeuwes, 2010a) are consistent with the notion that predictive remapping is a phenomenon that could underlie visual stability. 
However, remapping has also been used to refer to postsaccadic processes that are affected by the presaccadic allocation of visual attention ("memory remapping," Helcher, 2011; see also Szinte \& Cavanagh, 2011, 2012; Mathôt \& Theeuwes, 2010b; Collins, Rolfs, Deubel, \& Cavanagh, 2009; Golomb, Chun, \& Mazer, 2008; Bellebaum \& Daum, 2006; Bellebaum, Hoffmann, \& Daum, 2005). It has become increasingly clear that, although remapping may be initiated before the onset of an eye movement, there is a postsaccadic component: Visual stability is not yet fully restored immediately after a saccadic eye movement. More specifically, the focus of attention initially shifts, at least partly, with the eye movement (Mathôt \& Theeuwes, 2010a; Golomb et al., 2008; Blohm, Missal, \& Lefevre, 2005). This "retinotopic attentional trace" is quickly extinguished, but for a brief moment, the retinotopic nature of visual spatial attention can be exposed.

The first behavioral evidence for a retinotopic attentional trace was described by Posner and Cohen (1984). In one of their studies, participants made an eye movement, right after the brief presentation of a peripheral cue. A peripheral cue attracts attention, which can be measured as a faster response to subsequent stimuli presented at the same location. The crucial question was whether the locus of attention would move with the eyes (i.e., was retinotopic) or whether it would remain at the original cued location (i.e., was spatiotopic). Their results suggested that attention is purely retinotopic.

More recently, however, Golomb et al. (2008; see also Mathôt \& Theeuwes, 2010a) investigated the reference frame of visual attention in more detail. Their results added a significant new insight. Attention is retinotopic for a brief moment after an eye movement, in line with the results reported by Posner and Cohen (1984), but this is a transient phenomenon: After about $150 \mathrm{msec}$, the locus of attention is remapped back to its original location. The authors interpreted this finding as direct evidence for the existence of a transient retinotopic attentional trace. Golomb, Nguyen-Phuc, Mazer, McCarthy, and Chun (2010) followed up on this result by investigating the neural correlate of the retinotopic attentional trace using ERPs and fMRI. In their study, participants attended a fixed location in the center of a display, whereas distracting stimuli were presented briefly at various locations. The participants' task was to respond when a target stimulus (a stimulus that was slightly smaller than the distractors) appeared in the central location. This task served to maintain the participants' attention at the central location. Crucially, participants made an eye movement during the trial. After the eye movement, two types of locations could be distinguished. The "spatiotopic" location was the original, attended location in the center of the display. The "retinotopic" location was the location that, after the eye movement, occupied the same retinal position as the attended location had before the eye movement. ERPs to distractor stimuli presented at the retinotopic and spatiotopic locations were compared with a single control location. Importantly, Golomb, Nguyen-Phuc, et al. (2010) presented probe stimuli at two different intervals after the saccade, which allowed them to track the time course of the attentional remapping process. One major finding was that the P1, an ERP component that is known to be sensitive to spatial selective attention (Hillyard, Vogel, \& Luck, 1998), representing the suppression of the spatial selection at irrelevant item locations (Talsma, Mulckhuyse, Slagter, \& Theeuwes, 2007; Talsma, Slagter, Nieuwenhuis, Hage, \& Kok, 2005; Luck et al., 1994), was increased in amplitude for the long postsaccadic delay for both probe locations, compared with a control location. For the short postsaccadic delay, no clear P1 modulation was observed for either probe location, compared with the P1 components elicited by the control location.

In addition, Golomb, Nguyen-Phuc, et al. (2010) reported that the anterior N1, an ERP component believed either to reflect the cost of attending (Luck et al., 1994) or a response-related discrimination process (Vogel \& Luck, 2000), was modulated by stimuli presented at both the spatiotopic and the retinotopic location (compared with the control location), but only for the short postsaccadic delay. For longer postsaccadic delays, the anterior N1 was modulated only by stimuli presented at the spatiotopic location, compared with the control location. The finding that spatiotopic modulation of the anterior N1 was sustained, whereas retinotopic modulation was transient, compared with the N1 observed at the control location was interpreted as yet more evidence for a transient retinotopic attentional trace. Taken together, these results led the authors to conclude that two distinct loci of attention exist just after a saccade: a spatiotopic locus that is sustained and a transient, rapidly decaying retinotopic locus.

The aim of the present study was to expand upon the seminal findings of Golomb, Nguyen-Phuc, et al. (2010) by investigating the robustness of the retinotopic trace across a different paradigm. To do this, we used ERPs in a different paradigm that was similar to that of Golomb, Nguyen-Phuc, et al. (2010) but included an extra control location (cf. Mathôt \& Theeuwes, 2010a). This was done to address the potential confounding influence of "saccadic momentum" (Wang, Satel, Trappenberg, \& Klein, 2011). As shown in Figure 1B, relative to the fixation point following the saccade (Figure 1B; black arrow), the retinotopic location is roughly in the direction of the preceding eye movement $\left( \pm 45^{\circ}\right.$ angle; Figure $1 \mathrm{~B}$ light gray arrows). In contrast, the spatiotopic location is roughly in the opposite direction $\left( \pm 135^{\circ}\right.$ angle; Figure $1 \mathrm{~B}$; dark gray arrows). Because it has been shown that it is easier for the oculomotor system (and plausibly the attentional system as well) to make two successive shifts in the same direction, as compared with opposite directions, separate control locations were included to provide separate baselines for both the spatiotopic and the retinotopic locations.

In addition, behavioral data suggest that, just after a saccade, the locus of attention is predominantly retinotopic (Mathôt \& Theeuwes, 2010b; Golomb et al., 2008; 


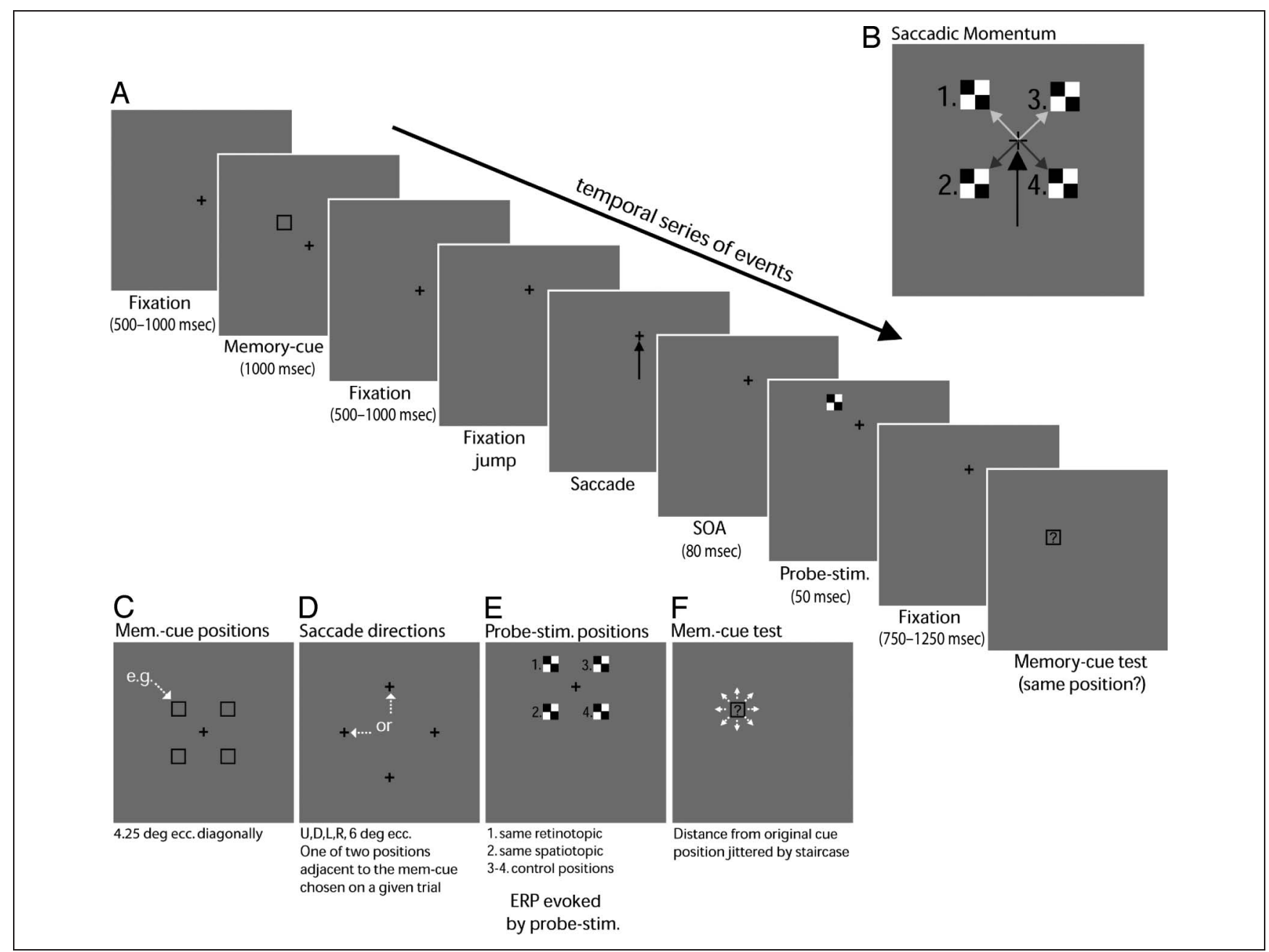

Figure 1. Outline of the paradigm. (A) Sequence of events on a given trial. A central fixation cross was presented for $1000 \mathrm{msec}$ after which a memory cue stimulus appeared for $1000 \mathrm{msec}$ at one of four possible locations. (B) Illustration of saccadic momentum. After participants ended a saccade, two probe positions are located more or less ahead of the saccadic trajectory (light gray arrows), whereas the other two positions lie behind the saccadic trajectory (dark gray arrows). (C) Layout of the four possible positions to be memorized. (D) After the memory cue disappeared, participants were required to hold fixation for an additional 500-1000 msec until the fixation point moved to a new location. Participants launched a saccade to the new location, which was constrained to the two possibilities adjacent to the memory cue on a given trial (D). (E) The gaze contingent probe stimulus appeared $(80 \mathrm{msec} \mathrm{SOA})$ at one of four possible locations illustrating the main conditions (see Methods). The probe was the critical event-related stimulus. (F) The memory cue test stimulus appeared with equal probability in either the same position as original memory cue or in a different position slightly jittered from its original location.

Posner \& Cohen, 1984). This is different from the ERP results reported by Golomb, Nguyen-Phuc, et al. (2010), who found that spatiotopic and retinotopic modulation of the anterior N1 was approximately equal for the shortest postsaccadic interval. Although this was interpreted as evidence for a lingering retinotopic trace, it is also possible that this reflects a diffuse spread of attention across both the retino- and spatiotopic regions immediately following the eye movement, which later becomes more focused on the goal-relevant spatiotopic region. Although Golomb, Marino, Chun, and Mazer (2010) provided behavioral evidence against such an explanation, critical evidence can be obtained by investigating the early latency visual ERP components that are sensitive to attentional manipulation, such as the exogenous posterior P1 and N1 components (Hillyard et al., 1998). Therefore, our main interest here was to determine whether these P1 and N1 components are modulated by stimuli presented at the retinotopic or spatiotopic locations, just after an eye movement. Specifically, given the association between P1 amplitude and visual attention (Hillyard et al., 1998), we hypothesized the presence of a strong P1 modulation for probes presented at the retinotopic location just after a saccade, and no or reduced P1 modulation for probes presented at the spatiotopic location.

\section{METHODS}

\section{Participants}

Thirteen healthy individuals (eight men, five women) took part in the study. All had normal or corrected-to-normal 
vision and ranged in age from 20 to 38 years. All participants gave written informed consent for their participation and were paid $€ 10$ per hour. One participant was excluded from the analyses due to an error in the data file containing the eye movement patterns.

\section{Apparatus}

The experiment was run in a dimly lit, sound-attenuated, electrically shielded cabin. Participants were seated facing the video display, with their heads stabilized by a chin rest. Stimuli were presented on a 21-in. CRT monitor at a screen resolution of $1024 \times 768$ pixels, running at a refresh rate of $100 \mathrm{~Hz}$ (noninterlaced). The screen dimensions were $37 \mathrm{~cm} \times 29.6 \mathrm{~cm}$, and at a viewing distance of $68 \mathrm{~cm}$, this yielded a viewing angle of approximately $32^{\circ}$ horizontally and $26^{\circ}$ vertically. Stimulus presentation was controlled using a personal computer system running an in-house developed application for stimulus control, based on the Eyelink $\mathrm{C}++$ software development toolkit provided by the manufacturer of the eye-tracking equipment.

During the experiment, eye position was recorded using an Eyelink 1000 (SR Research Ltd., Mississauga, Ontario, Canada) eye-tracking system operating at a sampling frequency of $1000 \mathrm{~Hz}$. The Eyelink $1000 \mathrm{did}$ not require the traditional head-mounted cameras but instead used a camera system that was built into a chinforehead restraint device that stabilized the head. This allowed for a relatively easy integration with the EEG cap system.

EEG signals were recorded with 128 active sintered Ag-AgCl electrodes (BioSemi, Amsterdam, The Netherlands), positioned radially equidistant from the vertex across the scalp (BioSemi 128 channel ABCD layout). Additional electrodes were placed at the left and right mastoids, approximately $1 \mathrm{~cm}$ above and below the orbital ridge of each eye, and at the outer canthi of the eyes. Eye movements were also monitored using a closed-circuit video system for on-line monitoring by the experimenter. Data were digitized at $512 \mathrm{~Hz}$ and referenced during recording to an active common mode electrode and rereferenced off-line to average of the two mastoids.

In addition to using the eye tracker, horizontal eye movements were detected by rereferencing the electrodes at the left and right canthi against each other, thus reconstructing the horizontal EOG. Likewise, vertical eye movements and blinks were detected by referencing the electrodes above each eye against the electrode directly below it. This was done for each eye separately, thus reconstructing the vertical EOGs. The purpose of the EOG recordings was to deconvolve the EEG recordings, thus to remove the artifact resulting from the task-induced eye movements.

\section{Experimental Design and Stimuli}

Figure 1A shows the sequence of events on a given trial. All stimuli were presented on a neutral gray background.
Initially, a central fixation cross $\left(0.5^{\circ}\right.$ in diameter $)$ was presented for a fixed duration of $1000 \mathrm{msec}$ after which a memory cue stimulus (consisting of a $1^{\circ} \times 1^{\circ}$ black square outline) appeared for $1000 \mathrm{msec}$ at one of four possible locations $4.25^{\circ}$ eccentricity at $45^{\circ}$ (angular) to one side or the other of the upcoming saccade trajectory (example location highlighted in Figure 1C). Participants were required to keep their eyes fixated at the central cross and to keep the precise location of the cue in memory for the duration of the trial. After the memory cue disappeared, participants were required to hold fixation for an additional 500-1000 msec until the fixation point moved to a new location. Then, participants were required to make a saccadic eye movement to that new location. There were four possible saccade directions (Figure 1D), which on a given trial were constrained to the two possibilities adjacent to the memory cue. This constraint was introduced to ensure that, after completion of the saccade, the memorized location remained at a visual angle that was of the same magnitude before and after the saccade. In the example in Figure 1, the top left memory cue was flashed, so the fixation cross could jump either upward or leftward, at an angular distance of $6^{\circ}$ eccentricity from the central location.

After the saccade to the new fixation location, a probe stimulus (a $2^{\circ} \times 2^{\circ}$ black and white checkerboard pattern also extending $1^{\circ} \times 1^{\circ}$ ) was presented at one of four possible locations (Figure 1E). The four possible probe stimulus locations are numbered in the example in which the fixation cross moved in an upward direction. As illustrated in Figure 1C, four functionally different probe stimuli were presented, based on their position in the display relative to the memory cue. Probe stimuli could be presented at (1) the same retinotopic coordinates as where the memory probe was presented (which after the saccade now corresponded to a different set of spatiotopic coordinates; henceforth referred to as the "retinotopic" probe location); (2) the same spatiotopic coordinates as where the memory cue was presented (now corresponding to a different set of retinotopic coordinates; henceforth referred to as the "spatiotopic" probe location); (3) a control position at a previously unattended location opposite the retinotopic location of the memory cue, perpendicular to the saccade trajectory (henceforth referred to as the "spatiotopic control" probe location); or (4) a control position that was also at an unattended location, but now placed at a location that was opposite the spatiotopic position, of the memory cue, also perpendicular to the saccade trajectory (henceforth referred to as the "retinotopic control" probe location). The precise timing of the appearance of this stimulus was gaze contingent. That is, the saccade had to be within $3^{\circ}$ of the new fixation location to trigger the appearance of the probe stimulus, which appeared $80 \mathrm{msec}$ from the time the eye first landed within this window. An SOA of $80 \mathrm{msec}$ was chosen because we were primarily interested in finding electrophysiological evidencce for the retinotopic 
attentional trace and Golomb et al. (2008) originally reported finding retinotopic effects at this SOA.

Lastly, a memory cue test stimulus appeared with equal probability in either the same position as original memory cue or in a different position slightly jittered from its original location (Figure 1F). Participants were required to respond with a key press whether it was in the same position or not. The difficulty of this task was controlled by varying the position of the memory cue test stimulus relative to the original using a staircase procedure with a 1-up 3-down rule aimed at yielding 79\% correct performance (Macmillan \& Creelman, 2005). The stimulus could be jittered randomly in one of eight possible directions ( $45^{\circ}$ apart; see Figure 1F), with a step-size distance of 10 pixels (corresponding to about $0.3^{\circ}$ of visual angle). The average step size across participants was 32 pixels or about $1^{\circ}$ of visual angle (range $=22-38$ pixels; $0.7-1.1^{\circ}$ of visual angle).

\section{Procedure}

Before the main experiment, participants performed a practice block to ensure they understood the requirements of the task. Upon successful completion of this practice block, the electrode caps were put in place and participants were again reminded of the task instructions before beginning the main experimental session. Each participant completed 10 blocks of 64 trials each, lasting a total of approximately $1.5 \mathrm{hr}$.

\section{Analyses}

\section{Behavioral Data}

Eye position was monitored throughout the experiment. Saccade-to-cue errors were defined as trials in which the eyes momentarily left central fixation and were directed toward the memory cue stimulus. The probe stimulus was triggered via a gaze contingent method: Gaze had to fall within a $3^{\circ} \times 3^{\circ}$ window around the new fixation location to trigger the probe stimulus. Occasionally the probe stimulus was not properly triggered, usually due to a loss in calibration accuracy. A recalibration was performed whenever this occurred. Furthermore, we applied additional off-line criteria to ensure that our analysis only included trials in which the gaze-contingent probe stimulus had been accurately triggered at the specified SOA of $\sim 80 \mathrm{msec}$. An off-line probe stimulus error was assigned if (1) gaze drifted beyond a $3^{\circ} \times 3^{\circ}$ window around central fixation from -500 to $-200 \mathrm{msec}$ relative to probe onset or (2) gaze fell outside a $3^{\circ} \times 3^{\circ}$ window around the new fixation location from 0 to $+200 \mathrm{msec}$ after probe stimulus onset. Finally, to confirm that participants encoded the location of the memory cue, performance on the memory cue task was analyzed to ensure that performance was maintained close to the ideal $79 \%$ across all participants (see the staircase procedure described earlier).

\section{ERP Data}

Raw EEG signals were digitally filtered using a 1.0-Hz, 4096-point finite impulse Gaussian high-pass filter and a 15-Hz, 1024-point finite impulse Gaussian low-pass filter. During digital filtering, recording artifacts causes by the task-induced eye movements, as well as eye blinks, were corrected using a frequency domain regression analysis (Kenemans, Molenaar, Verbaten, \& Slangen, 1991). Recording artifacts caused by other sources of interference were automatically discarded during the ERP averaging procedure, using an autoadaptive procedure that optimizes the signal-to-noise ratio in ERP data (Talsma, 2008). Of all trials, approximately $30 \%$ were discarded due to behavioral errors (i.e., a combination of behavioral errors on the memory task and incorrect eye movements). Of these behaviorally correct trials, approximately $30 \%$ were rejected due to artifacts. This left approximately 75-80 trials in each cell.

ERPs were obtained by time-locking to the onset of the probe stimulus and averaging all qualifying EEG epochs. These averages contained a $200-\mathrm{msec}$ prestimulus baseline period and extended until $1000 \mathrm{msec}$ after the event of interest. ERPs were baseline corrected using the $200 \mathrm{msec}$ prestimulus period. Separate ERPs were computed for each of the four above-described (retinotopic, spatiotopic, retinotopic control, and spatiotopic control) probe locations. Trials were collapsed across the four different saccade directions. For probes presented to the right visual hemifield, the positions of each corresponding left and right hemisphere electrode pairs were interchanged. This resulted in ERP and scalp topography plots where the right hemisphere represents activity contralateral to the location of the probe, whereas the left hemisphere represents activation ipsilateral to the probe (see Talsma et al., 2007, for a similar approach).

We initially tested each sample $(\sim 2 \mathrm{msec})$ and electrode for an effect of the within-subject factor Probe Location (i.e., spatiotopic, retinotopic, spatiotopic control, or retinotopic control position), using ANOVA. This analysis was limited to the first $500 \mathrm{msec}$ following stimulus onset. To reduce the probability of type I errors from occurring, we thresholded these results, such that effects were only considered to be significant when a $p<.05$ was observed for at least three sequential samples at two or more neighboring channels. These criteria were chosen because they represented the minimum temporal and spatial thresholds necessary to remove spurious results from our analyses (i.e., choosing more restrictive criteria did not change our results appreciably). After this overall analysis, two post hoc comparisons were conducted, in which specific levels of the factor Probe Location were tested against one of the other levels. More specifically, we conducted the following tests: (1) spatiotopic probe location versus spatiotopic control probe location and (2) retinotopic probe location versus retinotopic control probe location. Results from these tests were considered 


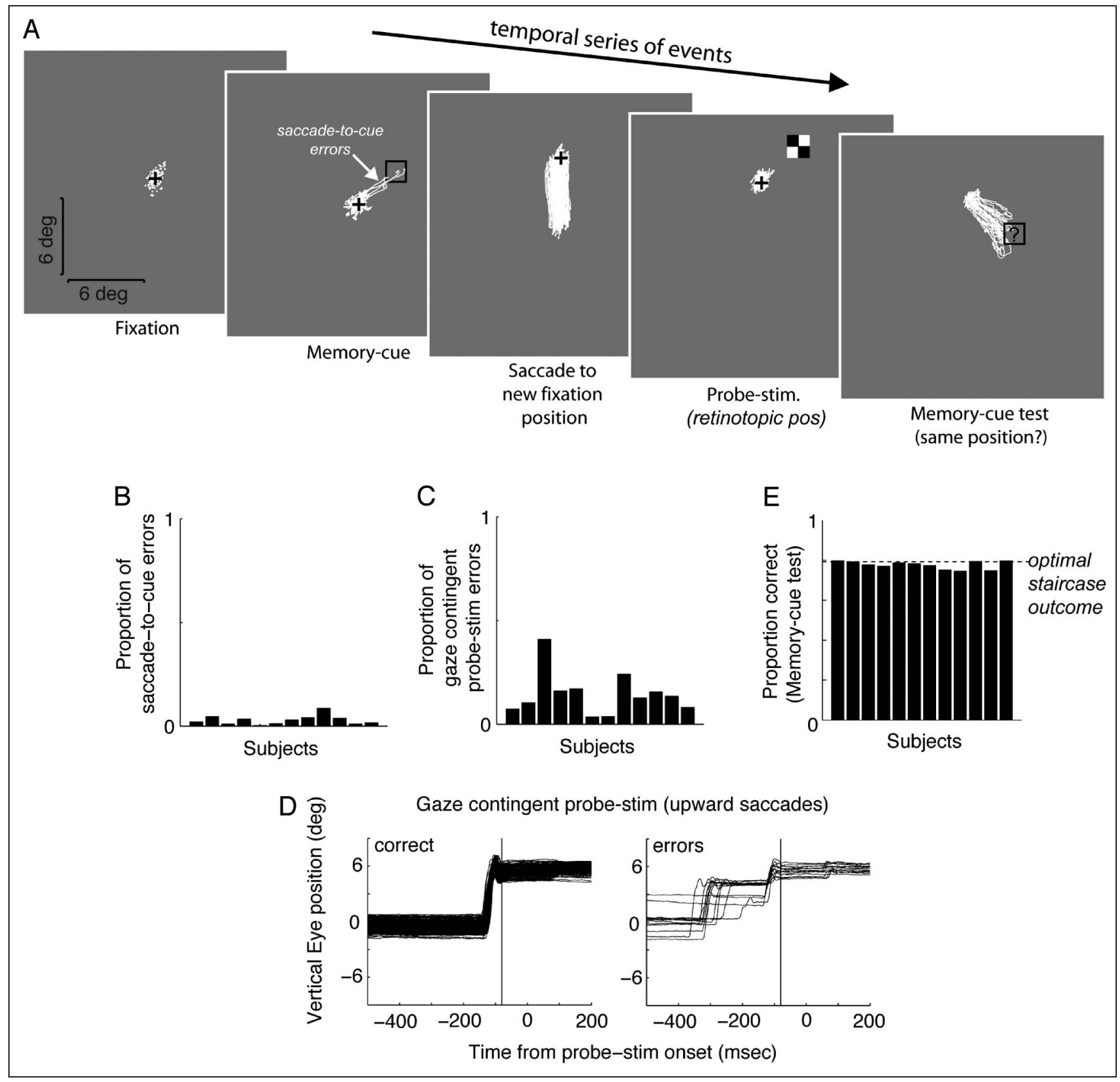

Figure 2. Behavioral data. (A) Example of the eye positions across the key events during a trial for one condition (several trials are illustrated). (B) Proportion of saccade-to-memory cue errors for each participant (examples illustrated in A). (C) Proportion of incorrectly triggered gaze contingent probe stimuli for each participant (example of upward directed saccades illustrated in D). A probe stimulus error was assigned if (1) eye position drifted beyond a $3^{\circ} \times 3^{\circ}$ window around central fixation from -500 to -200 msec relative to probe onset or (2) eye position was beyond a $3^{\circ} \times 3^{\circ}$ window around the new fixation location from 0 to $+200 \mathrm{msec}$ around probe stimulus onset. (E) Performance on the memory cue test for each participant.

significant when they adhered to the same criteria as applied to the overall test (i.e., $p<.05$ for at least three significant samples and for at least two adjacent channels), with the added restriction that any time point/ channel combination that had yielded no significant result on the overall test was discarded (cf. Van der Burg, Talsma, Olivers, Hickey, \& Theeuwes, 2011). Additionally, we calculated mean voltages across each cluster of electrodes/latency ranges for which our omnibus test had yielded a positive result. For each of these unique clusters, we then conducted four post hoc comparisons, using $t$ tests: (1) spatiotopic versus spatiotopic control, (2) retinotopic versus retinotopic control, (3) spatiotopic versus retinotopic, and (4) spatiotopic control versus retinotopic control. To prevent reporting false positives due to multiple comparisons, significance levels were adjusted by considering the false discovery rate (Benjamini \& Hochberg, 1995). 


\section{RESULTS}

\section{Behavioral Results}

Figure 2A shows an example of how gaze position changed across several key events during a given trial (several trials are illustrated). On a small proportion of the trials, the eyes were directed toward the memory cue (saccade-to-cue errors, Figure 2A, Panel 2). For all but one participant, less than $5 \%$ of trials were removed as a result of saccade-to-cue errors (Figure 2B; Participant 9 had 8.6\% saccade-to-cue errors). Saccades were then directed toward the new fixa- tion location (Figure 2A, Panel 3), after which the gaze contingent probe stimulus appeared. To ensure that our analysis only included trials in which the gaze-contingent probe stimulus was accurately triggered at the specified SOA of $\sim 80 \mathrm{msec}$, we plotted the eye position time-locked to the onset of the probe (see sample eye position in Figure 2D). Trials not meeting this criterion were automatically excluded from further analysis (see Behavioral Analyses in Methods). Figure 2D shows and sample of correct and error trials based on these criteria. The solid vertical line is centered at the ideal $80 \mathrm{msec}$ SOA. As can be seen from the

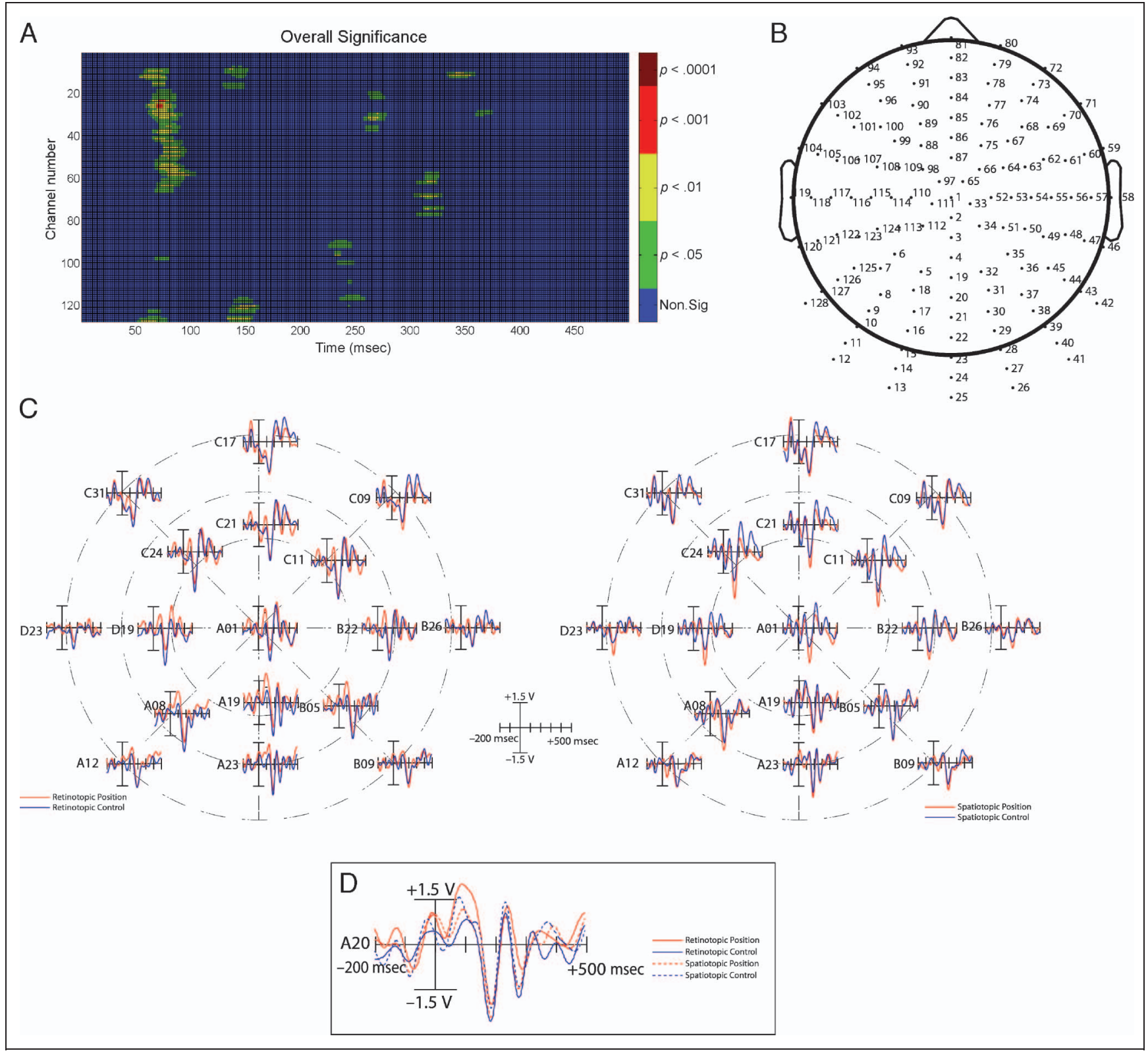

Figure 3. Summary overall statistics and electrode positions. (A) Shown here is the overall effect of Probe Location, across the four different probe positions (same retinotopic, same spatiotopic, retinotopic control, and spatiotopic control positions), as a function of time and electrode number, between 0 and $500 \mathrm{msec}$ after probe stimulus onset. (B) Electrode positions with corresponding channel numbers. Note that channel number corresponds to the BioSemi 128-channel ABCD layout (1-32 = A1-A32; 33-64 = B1-B32; 65-96 = C1-C32; and 97-128 = D1-D32; see www.biosemi.com for more details). (C) ERP waveforms time-locked to the onset of the probe stimuli, at a representative selection of electrodes. Illustrated are the ERPs elicited by the retinotopic probes versus their matched controls (top) and the ERPs elicited by the spatiotopic probes versus their matched controls (bottom). (D). Representative electrode contrasting ERPs in all four conditions. 


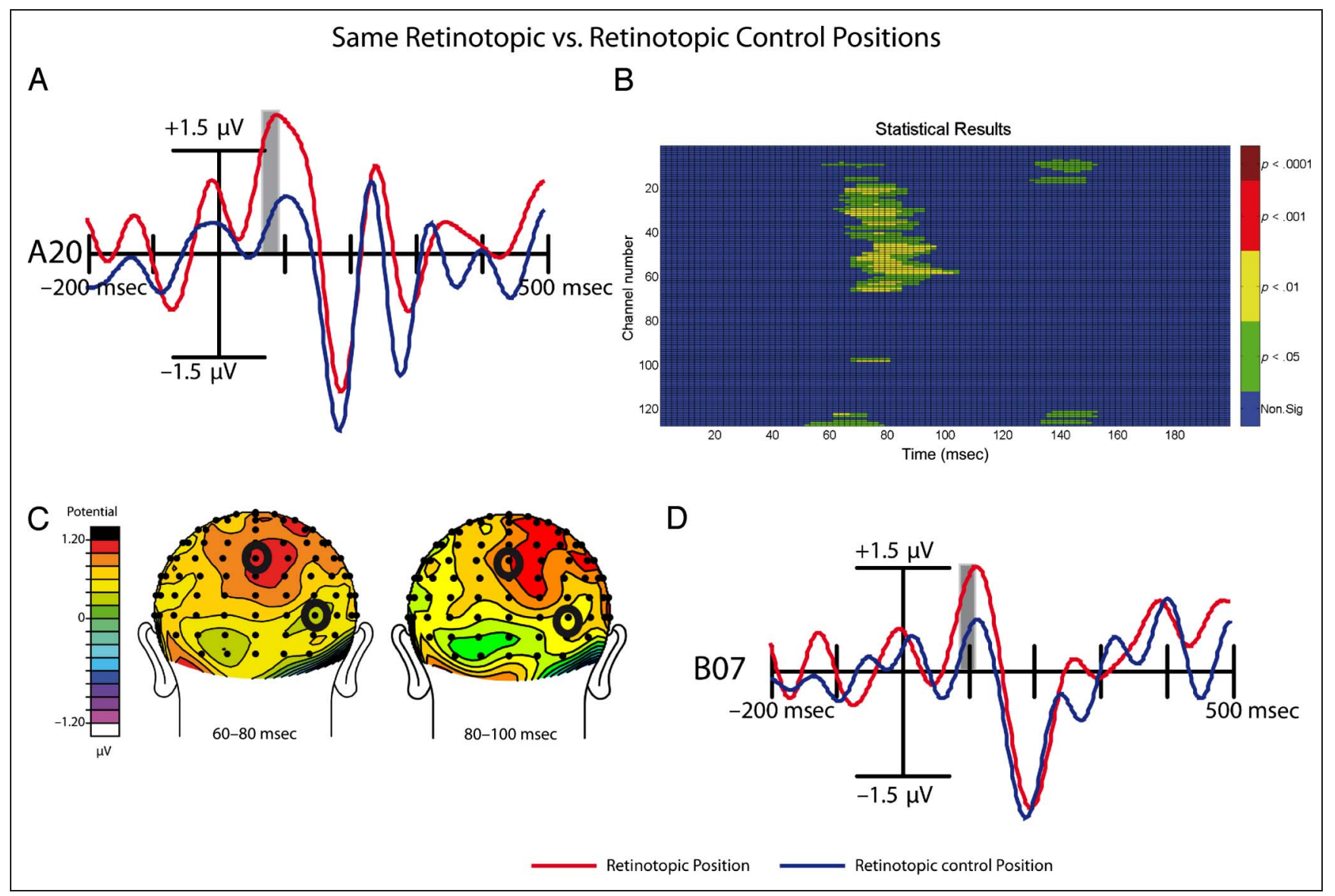

Figure 4. Same retinotopic versus retinotopic control positions. Shown here are the ERP results on two representative ERP channels (A and D), a plot of the channels and time windows where this contrast was significant (B), and the scalp distribution of the experimental effect, as obtained by subtracting the ERP activity obtained in the retinotopic control condition from the ERP activity obtained in the same retinotopic position (C).

sample of correctly triggered probe stimulus trials, the eye positions across all the trials were accurately aligned, with the end of the saccade very close to the ideal $80 \mathrm{msec}$ SOA. Across most subjects, the proportion of probe stimulus trigger errors was relatively low $(<17 \%$ except for Participants 3 and 8 , with $40 \%$ and $24 \%$, respectively), and these trials were removed to measure accurate probe-triggered ERPs. Lastly, Figure 2A (Panel 5) shows the eyes directed at the memory cue test stimulus. Recall that participants had to determine whether this stimulus was at the same or different location as the previous memory cue. Figure $2 \mathrm{E}$ shows the results of the memory cue task. Performance was accurately maintained between $74 \%$ and $79 \%$ ( $M=77 \%$ ) across participants (as a result of the staircase procedure, see Methods). This confirms that participants did indeed encode the location of the memory cue and that covert spatial attention was shifted in the direction of the memory cue.

\section{ERP Data}

\section{ERP Waveforms and Overall Effects}

Figure 3C shows the grand-averaged ERPs from a representative set of electrodes, time-locked to the onset of the probe stimuli. Over posterior recording sites, probe stimuli elicited the well-known sequence of P1 and N1 components. At anterior recording sites, the ERP waveforms were characterized by a relatively large frontally distributed N1 component. The N1 was followed in time by a sequence of P2, N2, and P3 components. It should be noted that the baseline period still contains some residual activity due to the relatively rapid sequence of events employed in the current paradigm. In spite of this, however, statistical analysis testing for the presence of a main effect of Probe Location (involving the ERPs elicited by all four probe stimulus location) revealed a sequence of several highly significant effects (Figure 3A), indicating that our experimental manipulations were strong enough. Most importantly, the posterior P1 ( 60-80 msec after probe onset) was different across these conditions, an effect that could be observed across a broad range of posterior electrodes. This effect was followed by a further sequence of statistically significant N1 ( 140-150 msec after probe onset), P2 ( $250 \mathrm{msec}$ after probe onset), and P3 ( 300-340 msec after probe onset) effects.

To test our hypothesis that attention-related neural sensitivity is coded in retinotopic coordinates immediately following an eye movement (as opposed to being 
due to saccadic momentum), we conducted two additional contrast analyses related to the early-latency P1 and N1 effects. More specifically, we contrasted the ERP responses elicited by the probe stimulus for the retinotopic versus the retinotopic control positions and the spatiotopic versus the spatiotopic control positions.

\section{Retinotopic versus Retinotopic Control Positions}

Figure 4 illustrates the main results of the retinotopic versus retinotopic control position contrast. Figure $4 \mathrm{~A}$ and D show waveforms recorded from the two sites highlighted in Figure 4C. The waveforms were aligned with the onset of the probe stimulus (red represents the retinotopic case, and blue represents to retinotopic control case; see Methods). One can see from these examples a clear divergence in the signal starting around 70-80 msec (Figure $4 \mathrm{~A}$ and $\mathrm{D}$, shaded areas) after the onset of the probe stimulus. Specifically, there was significantly greater activation when the probe appeared at the retinotopic location versus the retinotopic control location. Figure $4 \mathrm{~B}$ summarizes the statistical differences between these conditions across all channels. One can see from this plot that about half of the channels showed a similar significant difference between the retinotopic and retinotopic control location. This contrast became statistically significant at about 80-100 msec after stimulus onset and was most pronounced over the contralateral posterior recording sites. Closer inspection of this result revealed that these effects were significant at two clusters of electrodes: one cluster involving the medial parietal areas, whereas the second area was located over the contralateral occipital areas. Inspection of the ERP waveforms showed that this effect coincided with the P1 component and that this component was significantly larger when the probe appeared at the original retinotopic location, as compared with the retinotopic control location.

\section{Spatiotopic versus Spatiotopic Control Position}

Parallel to the retinotopic versus retinotopic control probe locations, we contrasted the ERP responses elicited by probe stimuli at the spatiotopic versus the spatiotopic control locations (see Methods). In contrast to the retinotopic versus retinotopic control contrast (Figure 4), this contrast did yield some evidence of a significant P1 effect

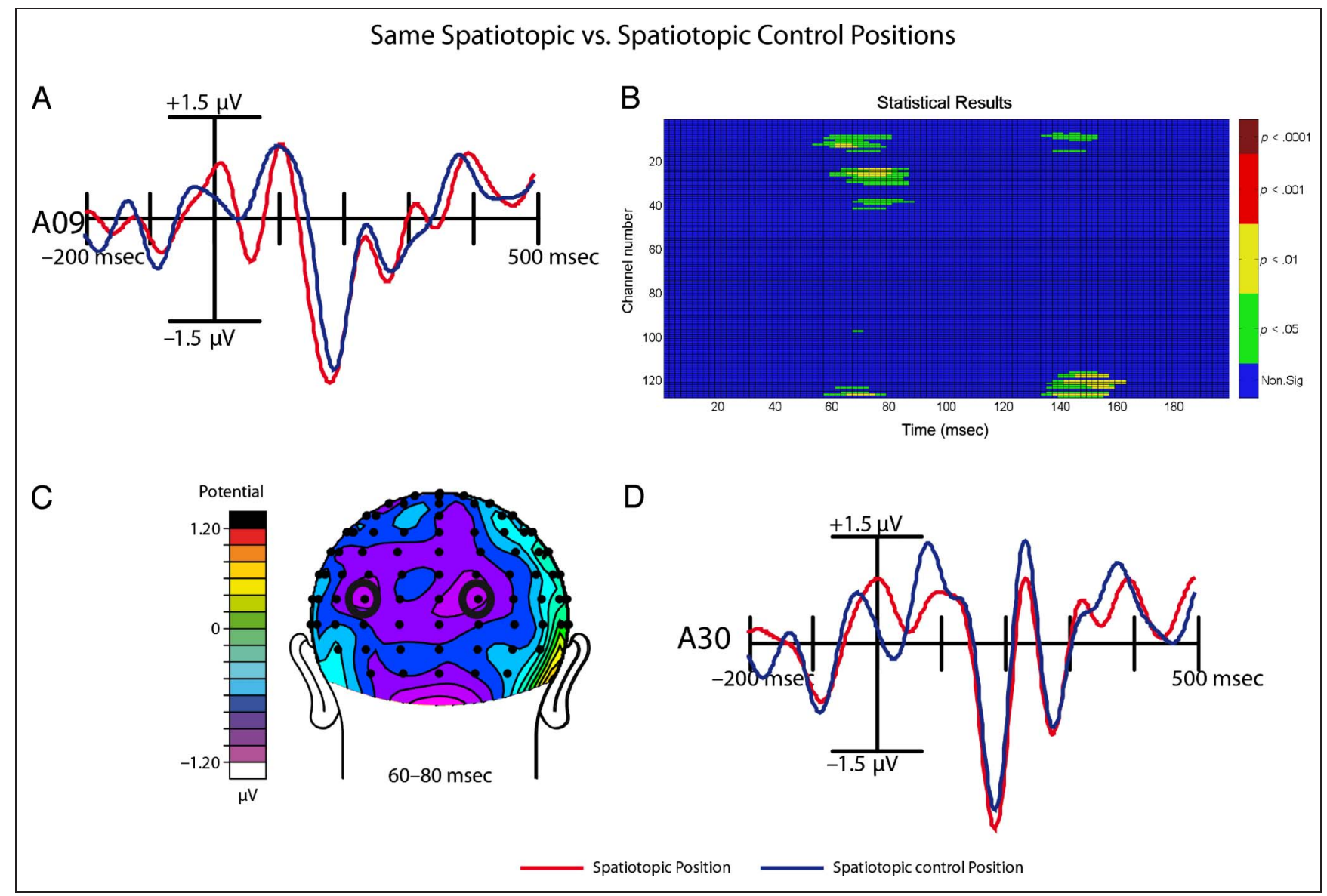

Figure 5. Same spatiotopic versus spatiotopic control positions. Shown here are the ERP results on two representative ERP channels (A and D), a plot of the channels and time windows where this contrast was significant (B), and the scalp distribution of the experimental effect, as obtained by subtracting the ERP activity obtained in the spatiotopic control condition from the ERP activity obtained in the same spatiotopic position (C). 
that was found over both the ipsi- and contralateral parietooccipital areas. However, the effect was significant across far fewer channels than in the previous contrast (see Figure 5B), and more importantly the effect was in the opposite direction compared with the retinotopic versus retinotopic control comparison: that is, the P1 was instead significantly smaller for the Spatiotopic probe condition than for the spatiotopic control condition.

\section{Longer Latency Frontal Effects}

As shown in Figure 6, several longer latency effects were present in the current data. Around $240 \mathrm{msec}$, ERPs elic-
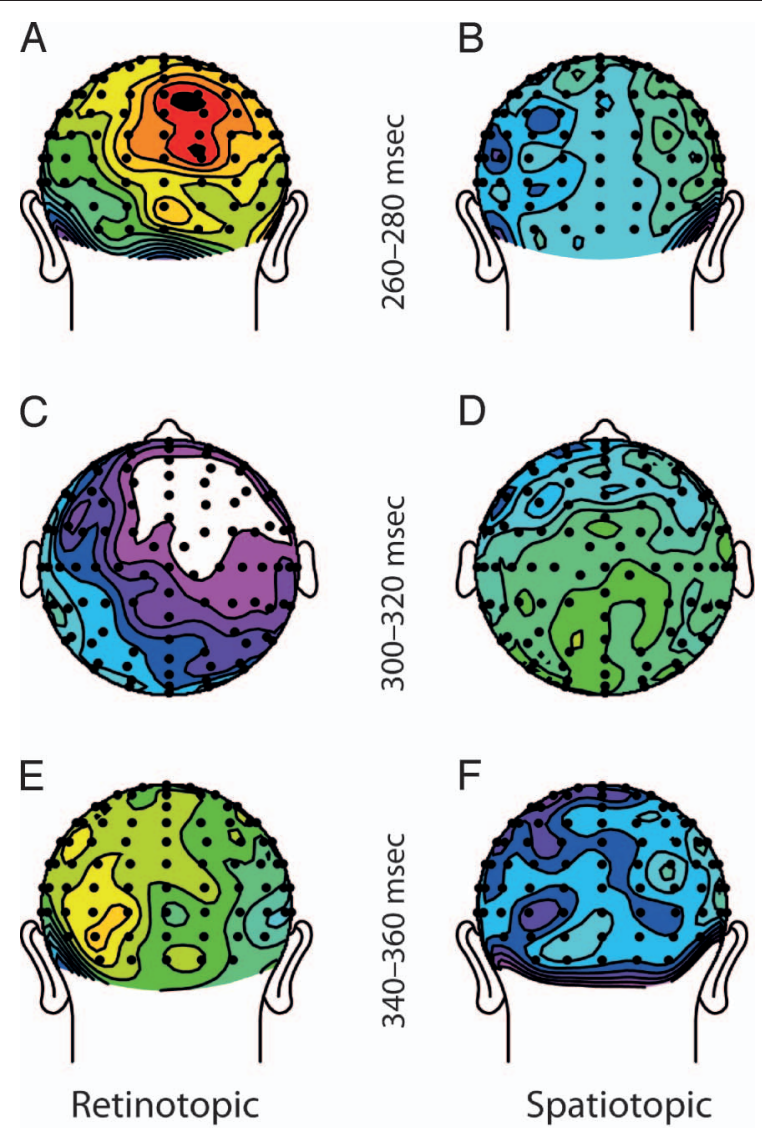

VS.

Matched control

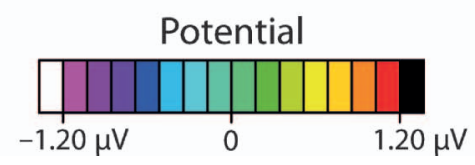

Figure 6. Longer latency effects. Shown here are the scalp topography plots for the two main contrasts of interests (spatiotopic vs. its matched control position and retinotopic vs. its matched control position) at three different latencies past the time windows of the initial P1 and N1 components. Top: contralateral parietal effect at 240-260 msec. Center: A fronto-central negative effect at approximated 300-320 msec. Bottom: An ipsilateral effect over occipital areas at around $350 \mathrm{msec}$. All latencies are relative to probe. Differences are the most pronounced for the retinotopic position versus retinotopic control conditions. ited by probes at the retinotopic versus retinotopic control position were characterized by a reduced N2 component over the posterior contralateral medial areas (Figure 6, top row). This effect was significant in the contrast between the retinotopic versus retinotopic control conditions between 260 and $280 \mathrm{msec}$ (Figure 6A) at electrodes B29-A32 (all ps <.01). For the contrast between the spatiotopic and spatiotopic control conditions (Figure 6B), this effect was not significant.

This N2 was followed by a fronto-central negative ERP when the probe stimulus appeared at the retinotopic location and was also characterized by a slight contralateral organization (Figure 6, center row). This effect, which was significant for the retinotopic versus retinotopic control contrasts (Figure 6C), lasted between 304 and $326 \mathrm{msec}$ (electrodes B25-B30); all $p s<.05$ ). It was not significant for the spatiotopic versus spatiotopic control conditions (Figure 6D). Finally, at around $340 \mathrm{msec}$ after stimulus, we observed an effect over the ipsilateral occipital electrodes (Figure 6, bottom row), which was significant between 336 and 360 msec after probe onset over the electrodes A11-A13. This effect was significant for both the retinotopic $(p<.05)$ versus retinotopic control contrast (Figure 6E; $p<.01)$ as well as for the spatiotopic $(p<.05)$ versus spatiotopic contrast (Figure $6 \mathrm{~F} ; p<.05$ ).

\section{Other Effects}

Finally, to obain a full idea of possible effects present within the clusters of electrodes and latency ranges that we identified as showing a significant effect, we computed mean voltages within each of these areas, for each condition separately, and subjected those mean voltages to pairwise $t$ tests. The result of this analysis is summarized in Table 1 . They largely confirm our analysis above, in the sense that most significant effects were found on the retinotopic versus retinotopic control contrast.

\section{DISCUSSION}

The current study investigated the neural representation of attentional processing immediately following a shift in gaze. Specifically, we compared visually elicited ERPs under different conditions designed to test the degree to which attention lingers in retinotopic coordinates immediately following a saccadic eye movement (Mathôt \& Theeuwes, 2010a; Golomb et al., 2008). In our experiment, participants were required to maintain visuospatial attention at a specific location, while subsequently executing a saccadic eye movement. Immediately following the saccade, a visual stimulus appeared at either at the original attended location (the "spatiotopic" location), a location that retinotopically matched the original attended location (the "retinotopic" location), or one of two control locations. The main result of the current study is that the P1 amplitude elicited by a probe stimulus was significantly 
Table 1. Student's $t$ Statistics for Post hoc Pairwise Contrasts

\begin{tabular}{|c|c|c|c|c|c|c|}
\hline Cluster & Electrode & $\begin{array}{l}\text { Latency } \\
\text { (msec) }\end{array}$ & $\begin{array}{c}\text { Spatiotopic vs. } \\
\text { Spatiotopic Control }\end{array}$ & $\begin{array}{c}\text { Retinotopic vs. } \\
\text { Retinotopic Control }\end{array}$ & $\begin{array}{l}\text { Spatiotopic vs. } \\
\text { Retinotopic }\end{array}$ & $\begin{array}{l}\text { Spatiotopic Control vs. } \\
\text { Retinotopic Control }\end{array}$ \\
\hline 1 & $7-12$ & $132-152$ & -2.0985 & 2.8584 & $-3.6218^{*}$ & 1.4623 \\
\hline 2 & $8-16$ & $54-80$ & -2.7252 & 2.4166 & $-3.1767 *$ & 1.3266 \\
\hline 3 & $10-12$ & $334-358$ & -1.6039 & 2.8129 & -2.7575 & 1.1873 \\
\hline 4 & $15-17$ & $130-148$ & -1.8055 & 2.6018 & -2.8462 & 1.0094 \\
\hline 5 & $18-66$ & $60-104$ & -1.5590 & $3.3469 *$ & -2.1049 & $2.9515^{*}$ \\
\hline 6 & $19-22$ & $262-276$ & -0.7391 & $3.0172 *$ & -2.7650 & 0.8369 \\
\hline 7 & $28-30$ & $360-374$ & -1.5311 & 1.8974 & -0.7333 & 2.7141 \\
\hline 8 & $29-32$ & $258-278$ & -0.4943 & $3.7937^{*}$ & -2.8265 & 1.0432 \\
\hline 9 & $35-37$ & $260-270$ & -0.0025 & $3.2981 *$ & $-3.2211^{*}$ & 0.2033 \\
\hline 10 & $57-62$ & $306-326$ & 0.2439 & $-3.4629 *$ & 1.1514 & -2.1948 \\
\hline 11 & $67-69$ & $304-328$ & -0.6921 & $-2.9607^{*}$ & 0.3387 & -2.1324 \\
\hline 12 & $73-74$ & $312-330$ & -0.4576 & $-2.9287 *$ & 0.1809 & -2.7884 \\
\hline 13 & $76-78$ & $306-328$ & -0.6844 & -2.6345 & 0.2786 & -2.2811 \\
\hline 14 & 89-92 & $226-246$ & -2.6764 & -0.6033 & -1.6751 & 0.9663 \\
\hline 15 & $94-96$ & $232-236$ & -2.6345 & -0.1667 & -1.5341 & 1.4649 \\
\hline 16 & $97-98$ & $68-80$ & -1.9995 & 2.6378 & -1.4050 & 2.3222 \\
\hline 17 & 99-100 & $234-248$ & -2.2933 & 0.6293 & $-3.1325^{*}$ & 0.8936 \\
\hline 18 & 108-109 & $244-246$ & -1.8252 & 1.2824 & $-2.9941^{*}$ & 0.7137 \\
\hline 19 & $115-117$ & $236-258$ & -2.0423 & 2.1538 & $-3.0098^{*}$ & 0.9270 \\
\hline 20 & $116-118$ & $140-156$ & -2.7528 & 1.8546 & -2.7717 & 1.3600 \\
\hline 21 & $120-123$ & $136-162$ & $-3.0744^{*}$ & 2.3517 & $-3.1830 *$ & 1.8716 \\
\hline 22 & $120-121$ & $244-244$ & -2.4064 & 1.4003 & -2.3812 & 1.6376 \\
\hline 23 & $122-123$ & $62-72$ & -2.1841 & $3.3212 *$ & -2.8283 & 1.2182 \\
\hline 24 & $125-128$ & $52-78$ & -1.8715 & $3.0029 *$ & $-3.6656 *$ & 0.4667 \\
\hline 25 & $125-128$ & $134-156$ & -2.6182 & 2.5646 & $-3.4758^{*}$ & 1.6346 \\
\hline
\end{tabular}

$t$ values were obtained by computing mean voltages for each of the significant clusters indicated in Figure 3. Values marked with a (*) are significant at $p=0.05$ after false discovery rate correction $(q=0.0114)$. For all tests, $d f=11$.

enhanced when this stimulus was presented at the retinotopic location, compared with when it was presented at the retinotopic control location. This effect was significant between 80 and 100 msec following the probe onset and was particularly isolated in contralateral occipital and medial parietal sites. Furthermore, when the probe was instead presented at the spatiotopic location (i.e., the original attended location), the visually evoked P1 was significantly lower compared with when the probe was presented at the spatiotopic control location. Taken together, these results are consistent with the hypothesis that there is a lingering retinotopic attentional trace immediately following a gaze shift: Right after an eye movement, attention is predominantly retinotopic (Mathôt \& Theeuwes, 2010a; Golomb et al., 2008).
Our results differ in some respects from those reported recently by Golomb, Nguyen-Phuc, et al. (2010). In their study, the probe stimuli did not elicit a significant P1 at a short SOA in any condition. Instead, they found a significantly enhanced P1 only at a much longer SOA (350-2250 msec), and it was the same for both the retinotopic and spatiotopic conditions. In addition, Golomb, Nguyen-Phuc et al. highlighted a later anterior N1 component (peak latency of 168-188 msec). This N1 was enhanced significantly for both the retinotopic and spatiotopic condition at the short SOA, but only for the spatiotopic condition at the long SOA.

Golomb et al. have discussed across several papers (Golomb, Marino, et al., 2010; Golomb, Nguyen-Phuc, et al., 2010; Golomb, Pulido, Albrecht, Chun, \& Mazer, 
2010; Golomb et al., 2008) that whereas the retinotopic trace persists regardless of task, spatiotopic facilitation is highly dependent on task relevance. For instance, in a retinotopic task (i.e., a task in which participants are explicitly instructed to maintain a location "relative to the eyes"; Golomb et al., 2008), spatiotopic facilitation was found to be nonexistent. In contrast, in tasks that are characterized by a much stronger spatiotopic emphasis (Golomb, Nguyen-Phuc, et al., 2010; Golomb, Pulido, et al., 2010), the spatiotopic facilitation could equal or exceed the retinotopic trace. For example, when participants were required to maintain attention on the spatiotopic location during the entire trial (Golomb, Nguyen-Phuc, et al., 2010) and to detect targets at this location, this could have created a much higher demand for ongoing spatiotopic attention than was the case in the present experiment, where one could argue that participants were only required to update the subsequent memory probe retrospectively.

The difference between our results and those of Golomb, Nguyen-Phuc, et al. (2010) could thus possibly be due to these differences in task-requirements. Our task was more similar to the original Golomb et al. (2008) experiment, and it should also be noted that our ERP results are consistent with the behavioral results of Golomb et al. (2008). Another difference between our approach and that of Golomb, Nguyen-Phuc, et al. is that we used behaviorally irrelevant probe stimuli to evoke an ERP response. The fact that there is no behavioral requirement to process these probe stimuli could have contributed to the fact that we only observed a retinotopic effect, whereas Golomb, Nguyen-Phuc, et al. observed ERP modulations in both the retinotopic and spatiotopic conditions. Thus, it appears that the retinotopic attentional trace is a robust phenomenon that has now been reported across a wide range of paradigms, and methods, whereas the spatiotopic counterpart depends more on specific task demands.

Related to the above-mentioned discrepancies, the manifestation of our retinotopic attentional trace differed somewhat from that of Golomb, Nguyen-Phuc, et al. (2010). Whereas in our study we predominantly observed major differences in the $\mathrm{P} 1$ latency range, the predominant effects observed by Golomb et al. were mainly reflected in a somewhat longer latency and more anteriorly distributed N1 component. It has been argued that the anterior N1 is sensitive to response discrimination processes (Vogel \& Luck, 2000). Therefore, it is much more likely that an anterior $\mathrm{N} 1$ is to be found in Golomb, Nguyhen-Phuc et al.'s experiment; they required participants to attend to one central location, where they were required to discriminate between target and non-target stimuli, whereas in our experiment, participants were not required to respond to the probe stimuli that we used to evoke an ERP response (as discussed above).

Another possibility is that, in line with Golomb, NguyhenPhuc, et al.'s observation, spatiotopic trace develops some- what later. Because the current study specifically focused on investigating the robustness of retinotopic attentional trace, we opted to present stimuli only at a fixed interval of $80 \mathrm{msec}$ after the end of the saccade. This time window was chosen on the basis of previous studies that reported retinotopic effects at this SOA (Golomb et al., 2008) but leaves out the possibility of investigating effects at later SOAs.

Taken together, it appears that the effects that we observed are driven mainly in a pure visual bottom-up fashion, whereas the effects reported by Golomb, NguyenPhuc, et al. are governed by a combination of both perceptual and task demands. Perhaps this distinction can also explain why we observed an effect in the P1 latency range, whereas Golomb, Nguyen-Phuc, et al. predominantly observed their main effects at a later stage. Because the P1 attention effect is considered to reflect the suppression of irrelevant information (Luck et al., 1994), our P1 effect could indicate that our specific task yielded a very strong initial suppression of the unattended locations, which then translated into an initial lower perceptual sensitivity for the processing of any spatial location that does not correspond with the original spatial location. Although further research is clearly needed to uncover the exact processes underlying this effect, we tentatively propose that this may be due to the requirement of keeping a strong attentional focus that is needed to memorize the exact position of the attended location.

It should be noted that our P1 effects are somewhat atypical in the sense that they occur relatively early and that the scalp distribution is somewhat more parietal and superior than the typical P1 components usually reported in the literature. There are number of possible reasons for this. First, our ERP evoking probe stimulus was presented shortly after the end of a saccade. Because there is presumably a suppression of visual input around the time of saccades (Burr, Morrone, \& Ross, 1994), this might play a role in meditating the timing of visual signals that elicited the ERP with this experimental design. Second, our probe stimuli were embedded in a relatively rapid sequence of visual stimuli. Previous ERP studies using relatively rapid visual sequences (Van der Burg et al., 2011; Leblanc, Prime, \& Jolicœur, 2008; Martens, Munneke, Smid, \& Johnson, 2006) have suggested that P1 activity is suppressed during such rapid sequences. Third, it should be noted that in our study we collapsed across all four possible stimulus locations, thereby effectively collapsing across upper and lower visual field probe stimuli. Given that attention effects on the P1, specifically at longer latencies, can reverse polarity between upper and lower visual field stimuli (Di Russo, Martínez, \& Hillyard, 2003), it is possible that these effects have limited our ability to observe the somewhat longer latency and more occipitally distributed effects. Fourth, it is possible that our early effects were somewhat attenuated by the fact that probe stimuli in one hemisphere were always preceded by saccades in one direction for one probe type and by saccades in the opposite 
direction for the other probe type. For instance, across conditions the retinotopic probes in the left visual field would be preceded by one upward saccade, one downward saccade, and two leftward saccades whereas spatiotopic probes in the same hemisphere would be preceded by one downward saccade, one upward saccade, and two rightward saccades. Although these factors may have contributed to the overall results, it should be stressed that this difference cannot explain our overall pattern of results, because if that had been the case, we would also have expected to find very similar effects between our conditions of interest and our control conditions, which is clearly not the case.

Finally, we could entertain the possibility that the ERP component that we interpret is actually a somewhat delayed C1 component. Although both the scalp topography and onset latency would be consistent with such an interpretation (Di Russo et al., 2003), it is typically assumed that the C1 component is not sensitive to manipulations of attention (Martínez et al., 1999; Hillyard \& Anllo-Vento, 1998), although some notable exceptions have recently been reported in the literature (Rauss, Pourtois, Vuilleumier, \& Schwartz, 2009; Kelly, GomezRamirez, \& Foxe, 2008). Although this leaves open the possibility that attention may influence the C1 amplitude, it should be noted that the findings of Kelly et al. were obtained by tailoring the stimulus presentation protocol to the spatial positions that were optimal for C1 evocation. Because this was not done in the current study, where we took an almost opposite approach of creating conditions that were clearly suboptimal for C1 evocation, we still tentatively interpret our findings in terms of a P1.

Adding to these early latency effects, we found a number of longer latency effects. Specifically, starting at around $260 \mathrm{msec}$ after stimulus onset, the N2 component was reduced in amplitude for probes presented at the retinotopic location, compared with the retinotopic control location. This finding is particularly interesting because it is generally enhancement, rather than attenuation, of the parietal N2 component that is associated with increased attentional processing (Johannes, Münte, Heinze, \& Mangun, 1995). Although we are currently unable to determine what this effect represents, an intriguing possibility is that it reflects a remapping process, in which top-down processes actively suppress the initial bottom-up driven retinotopic attentional trace. This putative mechanism could serve to avoid maladaptive persistent retinotopy. Future studies, designed specifically to address this question are required to elucidate this question.

Finally, it should be noted that there are some interesting similarities between our current results and results in other domains in the literature. For instance, in the domain of sensory motor integration, it appears that both perceptual (Gottlieb, Kusunoki, \& Goldberg, 1998) and motor commands (Buchholz, Jensen, \& Medendorp, 2011; Medendorp, 2011; Van Pelt \& Medendorp, 2008) are encoded in a retinotopic coordinate system. Although this suggests the existence of a common mechanism that is involved in updating this coordinate system, the subtle timing differences observed between the low-level neural processes involved in anticipatory shifting receptive fields in the perceptual system (Kusunoki, 2003) and the currently emerging evidence of a retinotopic attentional trace suggest a more complicated interaction than previously hypothesized. This notion would be consistent with recent work (Howe, Drew, Pinto, \& Horowitz, 2011; Cavanagh, Hunt, Afraz, \& Rolfs, 2010), suggesting that attentional pointers act as an intermediary process in facilitating remapping at the neural level, suggesting that only a limited number of attended items are remapped.

In summary, the results of this study provide convincing evidence from ERPs, which corresponded closely to behavioral findings (Mathôt \& Theeuwes, 2010b; Golomb et al., 2008), that visuospatial attention is maintained in retinotopic coordinates immediately following gaze shifts. This was evidenced by a modulation in the P1 component elicited by a postsaccadic stimulus presented at various locations relative to a behaviorally relevant attended location. This P1 component could arise from early visual cortical areas where remapping is known to be weakest (e.g., V1-V2; Merriam, Genovese, \& Colby, 2007; Nakamura \& Colby, 2002). In other words, a retinotopic bias might dominate early in visual processing (e.g., V1-V2), whereas a spatiotopic bias indicative of remapping might arise later (e.g., extrastriate visual cortex, lateral intraparietal area).

\section{Acknowledgments}

This research was funded by grant 463-06-014 from the Netherlands Organization for Scientific Research (NWO) and grant HFSP-RGP39/2005 from the Human Frontiers Research Program to Jan Theeuwes and Douglas P. Munoz. We thank Paul Groot for software development.

Reprint requests should be sent to Durk Talsma, Department of Experimental Psychology, Faculty of Psychology and Educational Sciences, Ghent University, Henri Dunantlaan 2, 9000 Gent, Belgium, or via e-mail: durktals@gmail.com.

\section{REFERENCES}

Bellebaum, C., \& Daum, I. (2006). Time course of cross-hemispheric spatial updating in the human parietal cortex. Behavioural Brain Research, 169, 150-161.

Bellebaum, C., Hoffmann, K.-P., \& Daum, I. (2005). Post-saccadic updating of visual space in the posterior parietal cortex in humans. Behavioural Brain Research, 163, 194-203.

Benjamini, Y., \& Hochberg, Y. (1995). Controlling the false discovery rate: A practical and powerful approach to multiple testing. Journal of the Royal Statistical Society, Series B, Methodological, 57, 289-300.

Blohm, G., Missal, M., \& Lefevre, P. (2005). Processing of retinal and extraretinal signals for memory-guided saccades during smooth pursuit. Journal of Neurophysiology, 93, 1510. 
Buchholz, V. N., Jensen, O., \& Medendorp, W. P. (2011) Multiple reference frames in cortical oscillatory activity during tactile remapping for saccades. Journal of Neuroscience, 31, 16864-16871.

Burr, D. C., Morrone, M. C., \& Ross, J. (1994). Selective suppression of the magnocellular visual pathway during saccadic eye movements. Nature, 371, 511-513.

Cameron, B. D., Enns, J. T., Franks, I. M., \& Chua, R. (2009). The hand's automatic pilot can update visual information while the eye is in motion. Experimental Brain Research, 195, 445-454.

Cavanagh, P., Hunt, A. R., Afraz, A., \& Rolfs, M. (2010). Visual stability based on remapping of attention pointers. Trends in Cognitive Sciences, 14, 147-153.

Collins, T., Rolfs, M., Deubel, H., \& Cavanagh, P. (2009). Post-saccadic location judgments reveal remapping of saccade targets to non-foveal locations. Journal of Vision, 9, 1-9.

Di Russo, F., Martínez, A., \& Hillyard, S. A. (2003). Source analysis of event-related cortical activity during visuo-spatial attention. Cerebral Cortex, 13, 486-499.

Duhamel, J. R., Colby, C. L., \& Goldberg, M. E. (1992). The updating of the representation in visual space in parietal cortex by intended eye movements. Science, 255, 90-92.

Ferman, L., Collewijn, H., Jansen, T. C., \& Van Den Berg, A. V. (1987). Human gaze stability in the horizontal, vertical, and torsional direction during voluntary head movements, evaluated with a three-dimensional scleral induction coil technique. Vision Research, 27, 811-828.

Golomb, J. D., Chun, M. M., \& Mazer, J. A. (2008). The native coordinate system of spatial attention is retinotopic. Journal of Neuroscience, 28, 10654-10662.

Golomb, J. D., Marino, A. C., Chun, M. M., \& Mazer, J. A. (2010). Attention doesn't slide: Spatiotopic updating after eye movements instantiates a new, discrete attentional locus. Attention, Perception, \& Psychophysics, 73, 7-14.

Golomb, J. D., Nguyen-Phuc, A. Y., Mazer, J. A., McCarthy, G., \& Chun, M. M. (2010). Attentional facilitation throughout human visual cortex lingers in retinotopic coordinates after eye movements. Journal of Neuroscience, 30, 10493-10506.

Golomb, J. D., Pulido, V. Z., Albrecht, A. R., Chun, M. M., \& Mazer, J. A. (2010). Robustness of the retinotopic attentional trace after eye movements. Journal of Vision, 10, 1-12.

Gottlieb, J. P., Kusunoki, M., \& Goldberg, M. E. (1998). The representation of visual salience in monkey parietal cortex. Nature, 391, 481-484.

Grüsser, O. J. (1986). Interaction of efferent and afferent signals in visual perception: A history of ideas and experimental paradigms. Acta Psychologica, 63, 3-21.

Harrison, W. J., Mattingley, J. B., \& Remington, R. W. (2012). Pre-saccadic shifts of visual attention. PLoS One, 7, e45670.

Hillyard, S. A., \& Anllo-Vento, L. (1998). Event-related brain potentials in the study of visual selective attention. Proceedings of the National Academy of Sciences, U.S.A., 95, 781-787.

Hillyard, S. A., Vogel, E. K., \& Luck, S. J. (1998). Sensory gain control (amplification) as a mechanism of selective attention: Electrophysiological and neuroimaging evidence.

Philosophical Transactions of the Royal Society, Series B, Biological Sciences, 353, 1257-1270.

Howe, P. D. L., Drew, T., Pinto, Y., \& Horowitz, T. S. (2011). Remapping attention in multiple object tracking. Vision Research, 51, 489-495.

Hunt, A. R., \& Cavanagh, P. (2011). Remapped visual masking. Journal of Vision, 11, 1-8.

Johannes, S., Münte, T. F., Heinze, H. J., \& Mangun, G. R. (1995). Luminance and spatial attention effects on early visual processing. Cognitive Brain Research, 2, 189-205.
Kelly, S. P., Gomez-Ramirez, M., \& Foxe, J. J. (2008). Spatial attention modulates initial afferent activity in human primary visual cortex. Cerebral Cortex, 18, 2629-2636.

Kenemans, J. L., Molenaar, P. C. M., Verbaten, M. N., \& Slangen, J. L. (1991). Removal of the ocular artifact from the EEG: A comparison of time and frequency domain methods with simulated and real data. Psychophysiology, 28, 114-121.

Kusunoki, M. (2003). The time course of perisaccadic receptive field shifts in the lateral intraparietal area of the monkey. Journal of Neurophysiology, 89, 1519-1527.

Leblanc, E., Prime, D. J., \& Jolicœur, P. (2008). Tracking the location of visuospatial attention in a contingent capture paradigm. Journal of Cognitive Neuroscience, 20, 657-671.

Luck, S. J., Hillyard, S. A., Mouloua, M., Woldorff, M. G., Clark, V. P., \& Hawkins, H. L. (1994). Effects of spatial cuing on luminance detectability: Psychophysical and electrophysiological evidence for early selection. Journal of Experimental Psychology: Human Perception and Performance, 20, 887-904.

Macmillan, N. A., \& Creelman, C. D. (2005). Detection theory: A user's guide (2nd ed.). New York: Cambridge University Press.

Martens, S., Munneke, J., Smid, H., \& Johnson, A. (2006). Quick minds don't blink: Electrophysiological correlates of individual differences in attentional selection. Journal of Cognitive Neuroscience, 18, 1423-1438.

Martínez, A., Anllo-Vento, L., Sereno, M. I., Frank, L. R., Buxton, R. B., Dubowitz, D. J., et al. (1999). Involvement of striate and extrastriate visual cortical areas in spatial attention. Nature Neuroscience, 2, 364-369.

Mathôt, S., \& Theeuwes, J. (2010a). Evidence for the predictive remapping of visual attention. Experimental Brain Research, 200, 117-122

Mathôt, S., \& Theeuwes, J. (2010b). Gradual remapping results in early retinotopic and late spatiotopic inhibition of return. Psychological Science, 21, 1793-1798.

Medendorp, W. P. (2011). Spatial constancy mechanisms in motor control. Philosophical Transactions of the Royal Society, Series B, Biological Sciences, 366, 476-491.

Melcher, D. (2007). Predictive remapping of visual features precedes saccadic eye movements. Nature Neuroscience, 10, 903-907.

Melcher, D. (2011). Visual stability. Philosophical Transactions of the Royal Society, Series B, Biological Sciences, 366, 468-475.

Merriam, E. P., Genovese, C. R., \& Colby, C. L. (2007) Remapping in human visual cortex. Journal of Neurophysiology, 97, 1738-1755.

Murphy, B. J. (1978). Pattern thresholds for moving and stationary gratings during smooth eye movement. Vision Research, 18, 521-530.

Nakamura, K., \& Colby, C. L. (2002). Updating of the visual representation in monkey striate and extrastriate cortex during saccades. Proceedings of the National Academy of Sciences, U.S.A., 99, 4026-4031.

Parks, N. A., \& Corballis, P. M. (2008). Electrophysiological correlates of presaccadic remapping in humans. Psychophysiology, 45, 776-783.

Parks, N. A., \& Corballis, P. M. (2010). Human transsaccadic visual processing: Presaccadic remapping and postsaccadic updating. Neuropsychologia, 48, 3451-3458.

Posner, M. I., \& Cohen, Y. (1984). Components of visual orienting. Attention and Performance, 10, 531-556.

Rauss, K. S., Pourtois, G., Vuilleumier, P., \& Schwartz, S. (2009). Attentional load modifies early activity in human primary visual cortex. Human Brain Mapping, 30, $1723-1733$. 
Rolfs, M., Jonikaitis, D., Deubel, H., \& Cavanagh, P. (2011). Predictive remapping of attention across eye movements. Nature Neuroscience, 14, 252-256.

Slavenski, A. A., Hansen, R. M., Steinman, R. M., \& Winterson, B. J. (1979). Quality of retinal image stabilization during small natural and artificial body rotations in man. Vision Research, 19, 675-683.

Sommer, M. A., \& Wurtz, R. H. (2006). Influence of the thalamus on spatial visual processing in frontal cortex. Nature, 444, 374-377.

Szinte, M., \& Cavanagh, P. (2011). Spatiotopic apparent motion reveals local variations in space constancy. Journal of Vision, 11, 4-4.

Szinte, M., \& Cavanagh, P. (2012). Apparent motion from outside the visual field, retinotopic cortices may register extra-retinal positions. PloS One, 7, e45670.

Talsma, D. (2008). Auto-adaptive averaging: Detecting artifacts in event-related potential data using a fully automated procedure. Psychophysiology, 45, 216-228.

Talsma, D., Mulckhuyse, M., Slagter, H. A., \& Theeuwes, J. (2007). Faster, more intense! The relation between electrophysiological reflections of attentional orienting, sensory gain control, and speed of responding. Brain Research, 1178, 92-105.
Talsma, D., Slagter, H. A., Nieuwenhuis, S., Hage, J., \& Kok, A. (2005). The orienting of visuospatial attention: An eventrelated brain potential study. Cognitive Brain Research, $25,117-129$.

Van der Burg, E., Talsma, D., Olivers, C. N. L., Hickey, C., \& Theeuwes, J. (2011). Early multisensory interactions affect the competition among multiple visual objects. Neuroimage, 55, 1208-1218.

Van Pelt, S., \& Medendorp, W. P. (2008). Updating target distance across eye movements in depth. Journal of Neurophysiology, 99, 2281-2290.

Vogel, E. K., \& Luck, S. J. (2000). The visual N1 component as an index of a discrimination process. Psychophysiology, 37, 190-203.

Walls, G. L. (1962). The evolutionary history of eye movements. Vision Research, 2, 69-80.

Wang, Z., Satel, J., Trappenberg, T. P., \& Klein, R. M. (2011). Aftereffects of saccades explored in a dynamic neural field of the superior colliculus. Journal of Eye Movement Research, 4, 1-16.

Westheimer, G., \& McKee, S. P. (1975). Visual acuity in the presence of retinal-image motion. Journal of the Optical Society of America, 65, 847-850.

Wurtz, R. H. (2008). Neuronal mechanisms of visual stability. Vision Research, 48, 2070-2089. 